Communications in Physics, Vol. 18, No. 1 (2008), pp. 48-57

\title{
INFLUENCE OF SINTERING TEMPERATURE ON LOW-FIELD SPIN-POLARIZED TUNNELING MAGNETORESISTANCE OF $\mathrm{La}_{0.7} \mathrm{Ca}_{0.3} \mathrm{MnO}_{3}$
}

\author{
PHAM THANH PHONG \\ Ninh Hoa Department of Education and Training, Khanh Hoa, Vietnam \\ DO HUNG MANH, LE VAN HONG, NGUYEN XUAN PHUC \\ Institute of Material Science, VAST \\ NGUYEN VAN KHIEM, VU VAN HUNG \\ Department of Natural Sciences, Hong Duc University, \\ 307 Le Lai Str. Thanh Hoa City, Vietnam
}

\begin{abstract}
In this study we report the effect of sintering temperature on the low field magnetoresistance ( $L F M R$ ) of $\mathrm{La}_{0,7} \mathrm{Ca}_{0,3} \mathrm{MnO}_{3}$ manganite synthesized through sol-gel technique. The $\mathrm{La}_{0,7} \mathrm{Ca}_{0,3} \mathrm{MnO}_{3}$ has been sintered at $600{ }^{\circ} \mathrm{C}, 700^{\circ} \mathrm{C}, 800{ }^{\circ} \mathrm{C}, 900^{\circ} \mathrm{C}$ and $1200^{\circ} \mathrm{C}$. The crystallite as well as particle size also show strong dependence on the sintering temperature. While the ferromagnetic - paramagnetic (FM-PM) transition temperature remains almost constant, the metal - insulation transition temperature drop gradual and the low field magnetoresistance (LFMR) increase with a decrease in grain size. We have analyzed our data based on the spinpolarized transport of conduction electrons at the grain boundaries.
\end{abstract}

\section{INTRODUCTION}

The colossal magnetoresistance (CMR) in hole doped manganese oxides widely known as manganites with formula $\mathrm{L}_{1-x} \mathrm{~A}_{x} \mathrm{MnO}_{3}$, where $\mathrm{L}=\mathrm{La}, \mathrm{Nd}, \mathrm{Pr}$, etc and $\mathrm{A}=\mathrm{Ca}$, $\mathrm{Ba}, \mathrm{Sr}, \mathrm{Pb}$, etc., has been intensively studied over the last decade for their application potential [1]. This CMR (intrinsic MR) is usually observed around the PM-FM transition temperature $\left(\mathrm{T}_{C}\right)$ at a high magnetic field and is explained in terms of the Zener Double Exchange mechanism [2]. However, this model cannot properly explain all the details of observed CMR effect. Therefore, other theories have been developed, which besides DE mechanism also incorporate the Jahn-Teller character of $\mathrm{Mn}^{3+}$ ion by a variable electronphonon coupling [3]. The concept of phase separation has recently emerged according to which the physics of manganites in the CMR [1]. In polycrystalline samples, great values of LFMR (extrinsic MR) have been observed at temperatures well below $\mathrm{T}_{C}$ [4]. This extrinsic MR effect is dominated by spin polarized tunneling between neighboring grains $[4]$.

A number of such investigations of the grain size effect on electrical, magnetic, and magneto transport properties of perovskites $\mathrm{L}_{1-x} \mathrm{~A}_{x} \mathrm{MnO}_{3}$ have been recently published. Mahesh et al. [5] and Siwach et al. [6] have reported that in the grain size materials range 
of $25 \mathrm{~nm}-3.5 \mu \mathrm{m}$, the MR increases with decreasing grain size in the low temperature regime while the $\mathrm{MR}$ around $\mathrm{T}_{C}$ remains unaffected. In another report Sánchez et al. [7] show the MR be independent with grain size in the range $\sim 20 \mathrm{~nm}-110 \mathrm{~nm}$. Andrés et al. [8] proposed the concept of a conduction channel mechanism for polycrystalline manganites having grain size in the range of $12 \mathrm{~nm}-1,5 \mu \mathrm{m}$, based upon the nature of connectivity between grains. Later, Yuan et al. [9] discussed the transport phenomena for polycrystalline manganites in the light of spin polarized tunneling (SPT) model with a major consideration about the size of grain, which is essentially larger than $100 \mathrm{~nm}$ for their case. But the paper [9] does not clearly provide any physical explanation for gradual drop of the metal-insulator transition temperature $\left(\mathrm{T}_{p}\right)$ with decrease in grain size, while $\mathrm{T}_{C}$ remains almost constant. In this paper we studied detail the effect of sintering temperature on microstructure and low field magneto transport properties and based upon the SPT mechanism to give a plausible physical explanation of the observed electrical transport behavior over the whole temperature range studied (30-300K).

\section{EXPERIMENTAL}

A sol-gel method was used to prepared powder of $\mathrm{La}_{0.7} \mathrm{Ca}_{0.3} \mathrm{MnO}_{3}$ (LCMO). This method has the advantage of using low-temperature synthesis. Gel is then heated at a temperature $300^{\circ} \mathrm{C}$ for $2 \mathrm{~h}$. Phase pure completely crystalline samples have been obtained at the temperature as low as $600^{\circ} \mathrm{C}$. The LCMO samples were ground, pelletized and sintered at $\mathrm{T}_{S}=600,700,800,900$ and $1200^{\circ} \mathrm{C}$ for $6 \mathrm{~h}$ will hereafter be referred to as LC6, LC7, LC8, LC9 and LC12 respectively.

The structural characterization was done through X-ray diffraction (XRD) and surface morphology was observed by scanning electron microscope (SEM). The temperature dependent of resistivity, $R(T)$, and magnetoresistance of the samples were measured by a standard dc four-probe technique in the temperature range of $30-300 \mathrm{~K}$ and in applied magnetic field in the range $3 \mathrm{kOe}$. The magnetization of the samples was measured by a vibrating sample magnetometer (VSM).

\section{RESULTS AND DISCUSSION}

The crystalline and phase analysis of all the synthesized samples ( $\mathrm{S} 0$, S6, S7, S8, S9, S12) were determined by the powder X-ray diffraction and the corresponding pattern are shown in Fig.1a. All the samples are orthorhombic and single phasic. In the present solgel, the characteristic perovskite phase formation starts at significant low temperature of $600^{\circ} \mathrm{C}$ as compared to other conventional methods. The intensity of the X-ray peaks for the LCMO perovskite phase increases as sintering temperature $\left(\mathrm{T}_{S}\right)$ increases from $600{ }^{\circ} \mathrm{C}$ to $1000{ }^{\circ} \mathrm{C}$ indicating that the crystallinity of LCMO becomes better with higher sintering temperature. Fig. $1 \mathrm{~b}$ shows the reflection of the samples at the $2 \theta=32.8^{\circ}$. It is clear from figure that as the sintering temperature increases, the full width at half maximum (FWHM) decreases and hence the crystallite size increases.

The average crystallite sizes $\left(\mathrm{D}_{h k l}\right)$ of the samples are obtained by the X-ray line width using Scherrer formula $\mathrm{D}_{h k l}=0.89 \lambda[\beta \cos \theta]^{-1}$, where $\beta$ is the actual FWHM and $\theta$ is the 


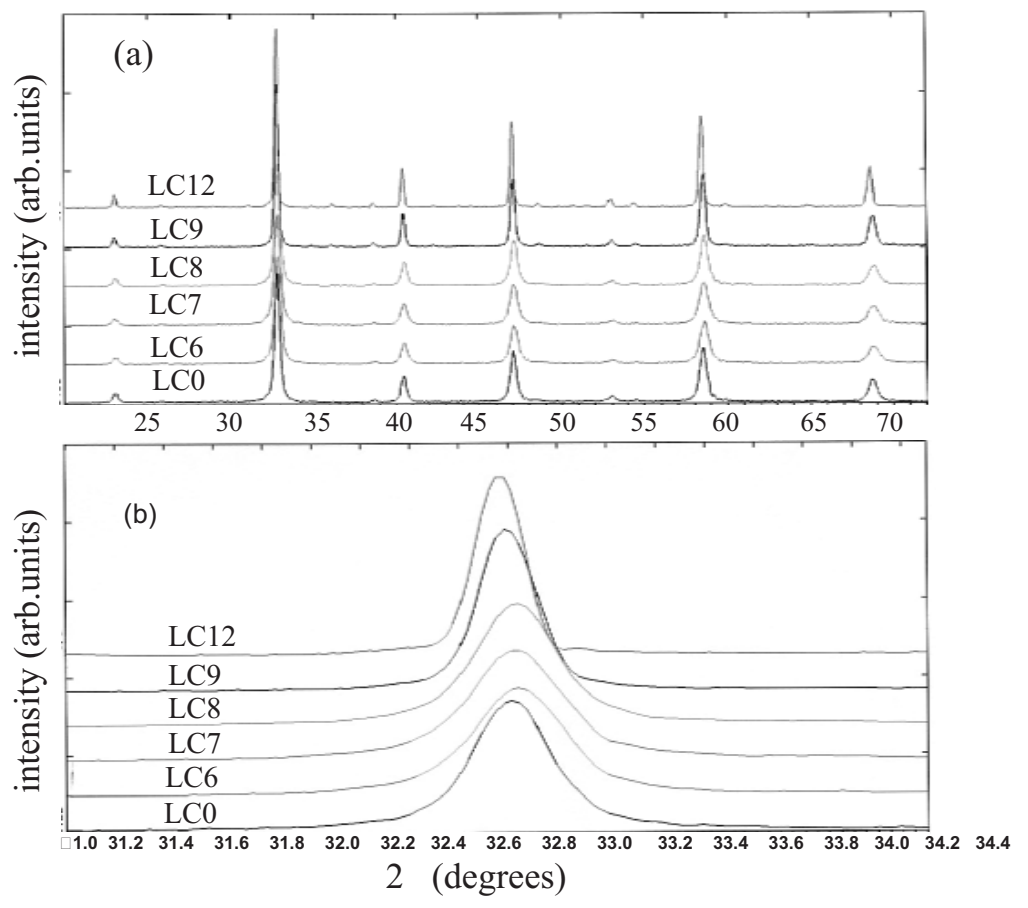

Fig. 1. (a) Powder X-ray Diffraction pattern of the as synthesized samples sintered at $200{ }^{\circ} \mathrm{C}(\mathrm{S} 0), 600{ }^{\circ} \mathrm{C}(\mathrm{S} 6), 700{ }^{\circ} \mathrm{C}(\mathrm{S} 7), 800{ }^{\circ} \mathrm{C}(\mathrm{S} 8), 900{ }^{\circ} \mathrm{C}(\mathrm{S} 9)$ and $1200^{\circ} \mathrm{C}(\mathrm{S} 12)$, (b) shows the width of the peaks for different sintered samples

angle of diffraction. The average crystallite size has been calculated to be $\sim 25,30,40$, 45, 60 and $75 \mathrm{~nm}$ respectively.

Fig. 2 shows the representative images elucidation surface morphology for the samples. SEM observation reveals that there is a distribution of particle size for all samples and as the sintering temperature increases, the particle size increases and the porosity decreases. The highest temperature $\left(1200^{\circ} \mathrm{C}\right)$ sintered sample (S12) has well connected particles whereas as we go down to lower temperature sintered sample, the particles connectivity becomes poor. The average particle size is to $32 \mathrm{~nm}$ from $250 \mathrm{~nm}$ for the samples LC0 and LC12, respectively. The crystallite sizes (CS) and the particle size (PS) obtained for the different samples are listed in Table 1. Both crystallite as well as particle size increase as the sintering temperature is increased due to congregation effect. However, it has been observed that there is a difference between CS and PS at all sintering temperature and is more pronounced at higher sintering temperature. For example, $\mathrm{CS}=30 \mathrm{~nm}$ and $\mathrm{PS}=$ $50 \mathrm{~nm}$ for LC6 and for S12 it is $75 \mathrm{~nm}$ and $250 \mathrm{~nm}$, respectively. This difference is due to the fact that particles are composed of several crystallites, probably due to the internal stress or defects in the structure [10].

The temperature dependence of magnetization (M-T) data were taken in the range 100 $-300 \mathrm{~K}$ (Fig. 3). $\mathrm{T}_{C}$ (is defined as the temperature corresponding to the peak of $d M / d T$ in the $M$ vs $T$ curse) is found about $265 \mathrm{~K}$ for all the samples. It has also been observed that 


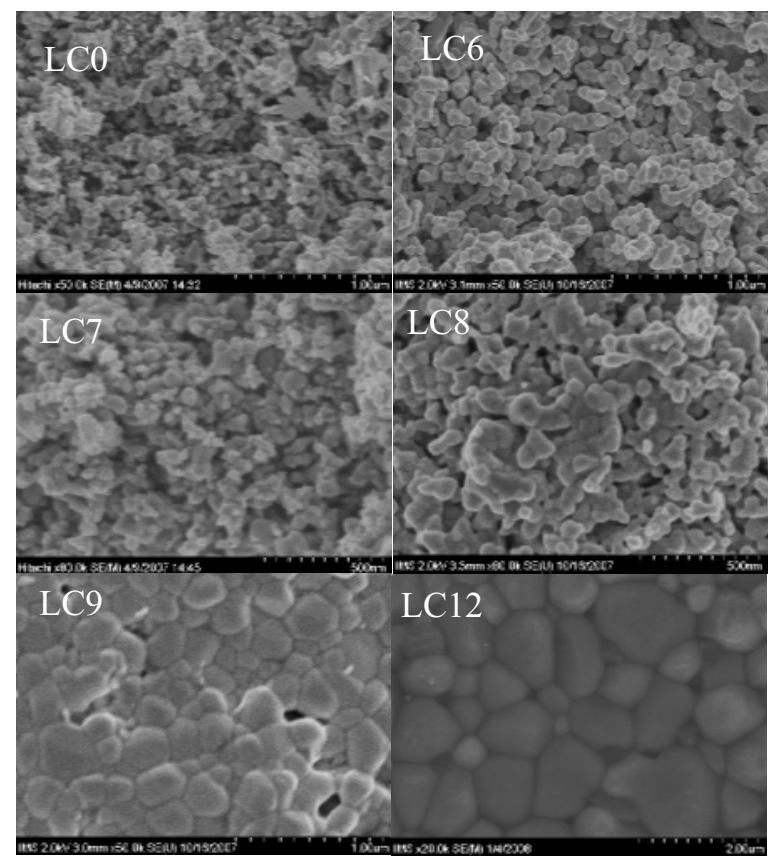

Fig. 2. SEM micrographs of the samples revealing surface morphology and particle size distribution

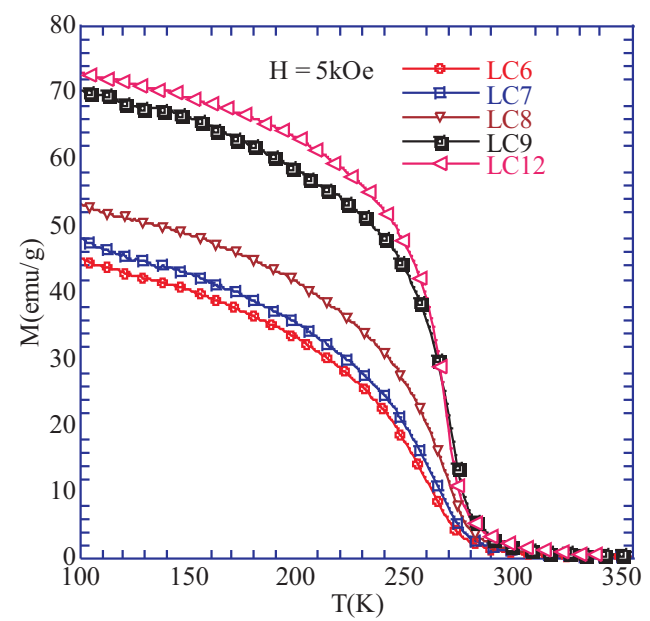

Fig. 3. Temperature dependence of magnetization measured at $5 \mathrm{kOe}$ for the samples sintered at different temperatures

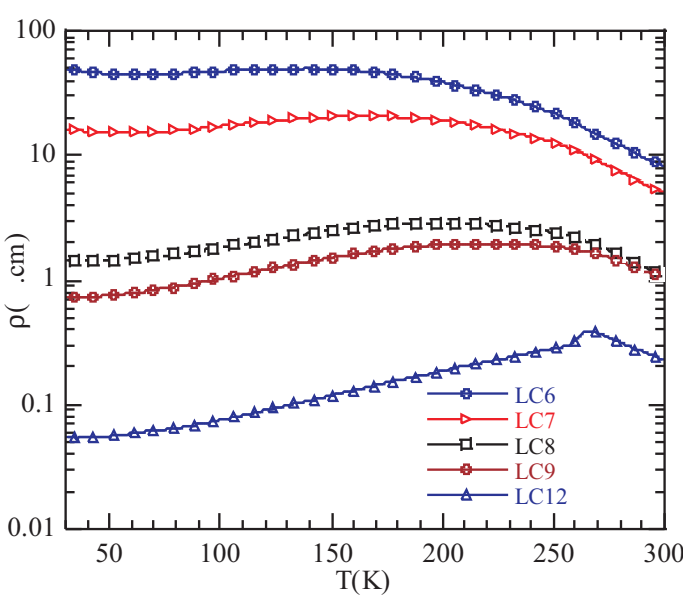

Fig. 4. Temperature dependence of resitivity $(\rho)$ of the samples sintered at different temperatures

as the sintering temperature decreases the width of transition broadens, which suggests that at low sintering temperature grains are loosely connected as also visible in the SEM 
shown in Fig. 2. Also Fig. 2 indicates that the magnetization of the samples increases as the sintering temperature increases. Which is same as found in earliest results [11].

The temperature dependence of resistivity was measured in the temperature range $\sim 30$ $300 \mathrm{~K}$. The dc resistivity $(\rho)$ of the LCMO samples exhibit strong dependence on the grain size. As the sintering temperature is decrease, the resistivity increase. This increase in resistivity is believed to be caused mainly due to enhanced scattering of the charge carriers by the higher density of magnetic disorder in grain boundaries (GBs) at smaller particle size. On increasing $\mathrm{T}_{S}$, the particle size increases leading to decrease in the GBs and the associated disorder. This results in decrease in scattering of the carriers expressed by a decrease in the resistivity.

All the samples show an increase in the resistivity on lowering temperature and at a characteristic temperature, which is lower than the corresponding $\mathrm{T}_{C}$, an insulator to metal like transition is observed. The insulator-metal transition temperature $\left(\mathrm{T}_{p}\right)$ are $160 \mathrm{~K}, 180 \mathrm{~K}, 220 \mathrm{~K}, 240 \mathrm{~K}$ and $265 \mathrm{~K}$, for LC6, LC7, LC8, LC9 and LC12, respectively. The insulator-metal transition temperature $\left(\mathrm{T}_{p}\right)$ obtained for the different samples are listed in Table 1.

Table 1. Crystalline size (XRD), particle size (SEM) and insulator-metal transition temperature $\left(T_{p}\right)$ of the samples sintered at different temperatures

\begin{tabular}{|c|c|c|c|}
\hline Sample & $\begin{array}{c}\text { Crystalline } \\
\text { size (nm) } \\
\text { XRD }\end{array}$ & $\begin{array}{c}\text { Particle } \\
\text { size (nm) } \\
\text { SEM }\end{array}$ & $\boldsymbol{T}_{p}(\mathbf{K})$ \\
\hline LC0 & 25 & 32 & \\
\hline LC6 & 30 & 50 & 130 \\
\hline LC7 & 40 & 65 & 165 \\
\hline LC8 & 45 & 80 & 198 \\
\hline LC9 & 60 & 150 & 217 \\
\hline LC12 & 85 & 300 & \\
\hline
\end{tabular}

The sol-gel prepared samples show a large difference between $\mathrm{T}_{C}$ and $\mathrm{T}_{p}$ and the difference increases as we lower the sintering temperature. The large difference in the $\mathrm{T}_{C}$ and $\mathrm{T}_{P}$ for all the LCMO samples is thought to be due to the existence of the disorder and is in fact a common feature of the polycrystalline maganites [12]. The $\mathrm{T}_{C}$ being an intrinsic characteristic does not show significant change as function of the sintering temperature. On the other hand $\mathrm{T}_{p}$ is an extrinsic property that strongly depends on the synthesis conditions and microstructure (e.g. grain boundary density).

Thus the $\mathrm{T}_{p}$ goes down by $135 \mathrm{~K}$ on lowering the sintering temperature from $1200{ }^{\circ} \mathrm{C}$ to $600{ }^{\circ} \mathrm{C}$ whereas $\mathrm{T}_{C}$ remains almost constant. The strong suppression of the $\mathrm{T}_{p}$ as compared to $\mathrm{T}_{C}$ is caused by the induced disorders and also by the increase in the non-magnetic phase fraction, which is due to enhanced grain boundary densities as consequence of lower sintering temperature. This also causes the increase in the carrier scattering leading to a corresponding enhancement in the resistivity. Thus lowering of sintering temperature reduces the metallic transition temperature and hence the concomitant increase in resistivity. 


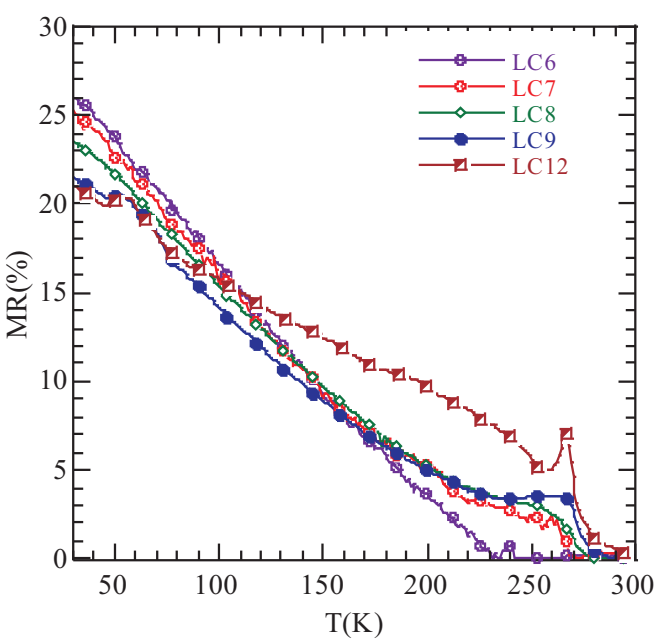

Fig. 5. Magnetoresistance (MR\%) as a function of temperature for applied magnetic field of $3 \mathrm{kOe}$ for the samples sintered at different temperatures

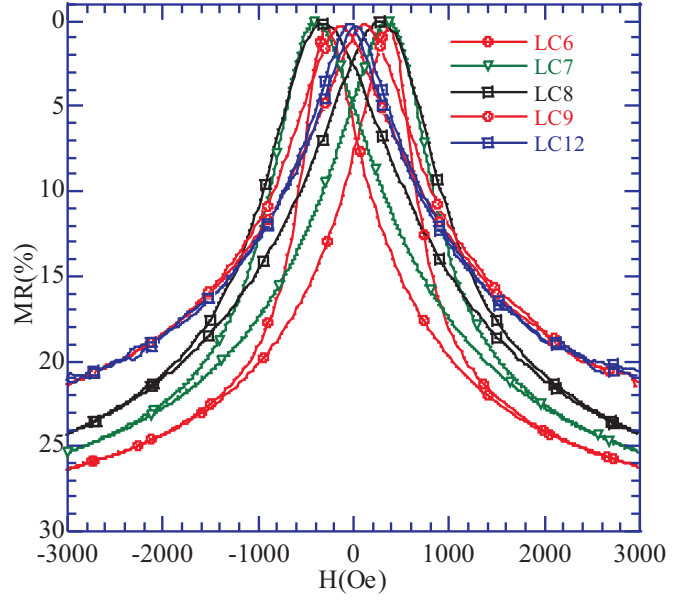

Fig. 6. Magnetoresistance (MR\%) as a function of magnetic field at $30 \mathrm{~K}$ for the samples sintered at different temperatures

The temperature dependence of MR (MR is calculated by the formula $\mathrm{MR}(\%)=\left[\left(\rho_{0}-\right.\right.$ $\left.\left.\rho_{H}\right) / \rho_{0}\right] \mathrm{x} 100$; where $\rho_{0}$ and $\rho_{H}$ are the resistivity measured at $\mathrm{H}=0$ and $\mathrm{H}$, respectively) for LC6, LC7, LC8, LC9 and LC12 samples measured in the range 30-300 K at 3kOe are shown in Fig. 5. All the samples show a sequential increase in low temperature MR with decreasing temperature. The appearance of peak in the (MR-T) curve around $\mathrm{T}_{C}$ depicts that in all the samples there is a contribution of the intrinsic component of MR, which arises due to the double exchange (DE) mechanism around $\mathrm{T}_{C}$. However, around $\mathrm{T}_{C}$ the peak in the (MR-T) curve of the sample LC12 is significantly higher in comparison to other samples. The peak MR values are $\sim 8 \%$ and $4 \%$ at $3 \mathrm{kOe}$ applied for sample LC9 and LC12 whereas for sample LC6, LC7, LC8 there is a hump in the MR variation around $\mathrm{T}_{C}$.

At $30 \mathrm{~K}$, the MR values are measured to be $\sim 26.36 \%, 25.35 \%, 24.29 \%, 21.49 \%$ and $20.67 \%$ for LC6, LC7, LC8, LC9 and LC12 respectively at the field of 3kOe (Fig. 6). Thus, decreasing crystalline/grain size leads to the enhancement in LFMR at lower temperatures while the MR in the higher temperature regime is suppressed. The disappearance of the high temperature MR can be explained by weakening of the DE mechanism around the respective FM - PM transition temperatures due to decrease in particle size which results from low sintering temperature. The LFMR increases as the sintering temperature and hence particle size decrease. This is consistent with previous studies $[10,11]$.

The magnetic field dependence at various temperatures of the LFMR of LC6 and LC7 are given in Fig.7. It can be observed that at $\mathrm{T}=30 \mathrm{~K}, \mathrm{LFMR}$ ( at $\mathrm{H}=3 \mathrm{kOe}$ ) is about $26 \%$ for the LC6 sample and $25 \%$ for the LC7 sample. In order to explore the basic physics behind this temperature dependence of MR in our nanocrystalline LCMO sample, our 
primary approach is to separate out the part of the MR originating from SPT $\left(\mathrm{MR}_{s p t}\right)$, from the part of the MR identified by the suppression of spin fluctuation $\left(\mathrm{MR}_{\text {int }}\right)$ and mainly to inspect their respective temperature dependencies. For this purpose, we have used the model as proposed by Raychaudhuri et al. [13] and Dey et al. [14], based on SPT transport of conduction electrons at the grain boundaries with attention paid to the magnetic domain wall motion at grain boundaries under the application of a magnetic field. According to this model we get the expression for MR as:
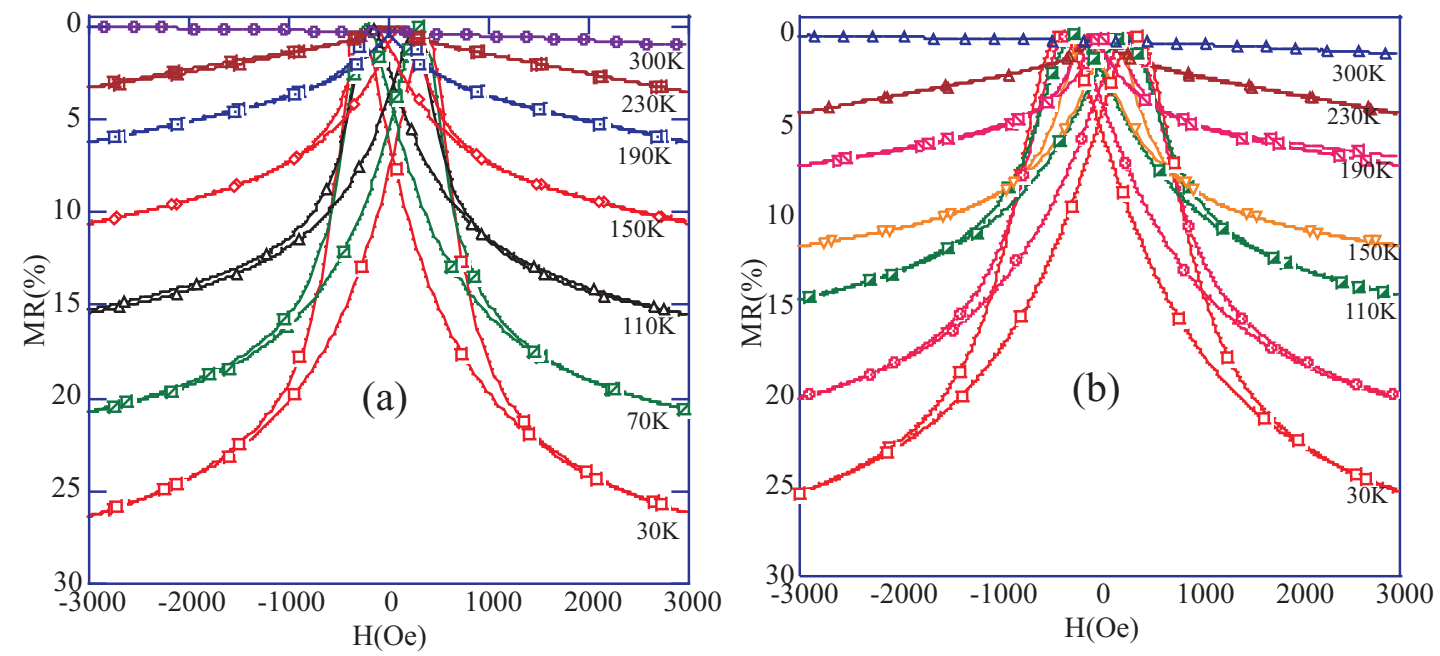

Fig. 7. Magnetoresistance (MR\%) as a function of magnetic field at various temperatures $(30-300 \mathrm{~K})$ for the sample sintered at $600^{\circ} \mathrm{C}(\mathrm{a})$ and $700^{\circ} \mathrm{C}(\mathrm{b})$

$$
M R=-A^{\prime} \int_{0}^{H} f(k) d k-J H-K H^{3}
$$

Within the approximation of the model, in zero field the domain boundaries are pinned at the grain boundary pinning centers having pinning strengths $k$. The grain boundaries have a distribution of pinning strengths (defined as the minimum field needed to overcome a particular pinning barrier) given by $f(k)$, expressed as:

$$
f(k)=A \exp \left(-B k^{2}\right)+C k^{2} \exp \left(-D k^{2}\right)
$$

All the adjustable fitting parameters, A, B, C, D, J, K with A' absorbed in A and C, are required to known from a nonlinear least square fitting to calculate $\mathrm{MR}_{s p t}$, which defined as:

$$
\mathrm{MR}_{\text {spt }}=-\int_{0}^{H} f(k) d k(3)
$$

Differentiating Eq. (1) with respect to $\mathrm{H}$ and putting Eq.(2), we get:

$$
\frac{d(M R)}{d H}=A \exp \left(-B H^{2}\right)+C H^{2} \exp \left(-D H^{2}\right)-J-3 k H^{2}
$$


Table 2. Experimental $M R$ (Expt. $M R), M R_{\text {spt }}(H), M R_{\text {int }}(H)$ at several temperature for nanocrystalline LCMO samples ( $L C 6$ and $L C 7$ ) sintered at different temperatures

\begin{tabular}{|c|c|c|c|c|}
\hline Sample & $\mathbf{T}(\mathbf{K})$ & $\begin{array}{c}\text { Expt.MR } \\
(\%)\end{array}$ & $\begin{array}{c}\mathbf{M R}_{\text {spt }} \\
(\%)\end{array}$ & $\begin{array}{c}\mathbf{M R}_{\text {int }} \\
(\%)\end{array}$ \\
\hline \multirow{5}{*}{ LC6 } & 30 & 26.36 & 22,76 & 3,60 \\
\cline { 2 - 5 } & 70 & 20.78 & 17.68 & 3.10 \\
\cline { 2 - 5 } & 110 & 15.45 & 12.54 & 2.91 \\
\cline { 2 - 5 } & 150 & 10.51 & 7.67 & 2.84 \\
\cline { 2 - 5 } & 190 & 6.27 & 3.11 & 3.16 \\
\cline { 2 - 5 } & 230 & 3.33 & 0.09 & 3.04 \\
\hline \multirow{5}{*}{ LC7 } & 30 & 25.25 & 20.04 & 5.21 \\
\cline { 2 - 5 } & 70 & 20.19 & 16.36 & 3.83 \\
\cline { 2 - 5 } & 110 & 14.43 & 11.29 & 3.14 \\
\cline { 2 - 5 } & 150 & 11.65 & 7.60 & 2.75 \\
\cline { 2 - 5 } & 190 & 6.72 & 3.33 & 3.39 \\
\cline { 2 - 5 } & 230 & 4.41 & 1.78 & 2.63 \\
\hline
\end{tabular}

The experimental (MR-H) curves were differentiated and fitted to Eq.(3) to find the best-fit parameters at several temperatures. Fig. 8 shows the differentiated curve and the best-fit function at $\mathrm{T}=30 \mathrm{~K}$ for LC6 sample and $\mathrm{LC} 7$ sample. The value of experimental $\mathrm{MR}, \mathrm{MR}_{\text {spt }}(\mathrm{H})$ and $\mathrm{MR}_{\text {int }}(\mathrm{H})$ at $\mathrm{H}=3 \mathrm{kOe}$ in Table 2 for nanocrystalline LC6 and LC7, respectively.

We observe that the total magnetoresistance is a no nmonotonic function of temperature with a slow decrease at low temperature followed by increases as we approach $\mathrm{T}_{C}$. The intrinsic contribution $\mathrm{MR}_{\text {int }}$, however, follows the expected $\mathrm{DE}$ behavior with a steady increase in temperature. On the other hand $\mathrm{MR}_{\text {spt }}$ de creases steadily with temperature. In order to elucidate the basic physics behind temperature dependence of MR, Dey et al. [14] believed that the nature of the surface region of nanosize grains plays a very crucial role in electrical transport, magnetic and magneto transport behavior of nanodimensional systems. When grain size of LCMO are $17 \mathrm{~nm}$ and $27 \mathrm{~nm}, \mathrm{MR}_{S P T}(\mathrm{H})$ remains constant up to a high temperature (about $\mathrm{T} \sim 200 \mathrm{~K}$ ) and then drops sharply with temperature. This effect gets enhanced with the decrease in particle size. This result for nanodimensional maganites is in contrast to the results reported by Hwang et al. [4] for $\mathrm{La}_{0,67} \mathrm{Sr}_{0,33} \mathrm{MnO}_{3}$ polycrystalline sample prepared through conventional solid-state reaction process in air and thus have a large grain size $(\sim \mu \mathrm{m})$. According to them the part of the MR most clearly identified with spin-polarized tunneling shows a gradual decrease with an increase in temperature. They had observed earlier that the temperature dependence of $\mathrm{MR}_{s p t}$ is described quite well by an expression of the type $a+b /(c+T)$, which is a characteristic of spin polarized tunneling in granular ferromagnetic systems.

Fig. 9 shows the best fit of $\mathrm{MR}_{s p t}$ with the expression $a+b /(c+T)$. The fitted curve matches well with the extracted values of $\mathrm{MR}_{\text {spt }}$ from model. However our values of $b$ and $c$ for the best fit are much higher compared to that observed by Hwang et al. although the 

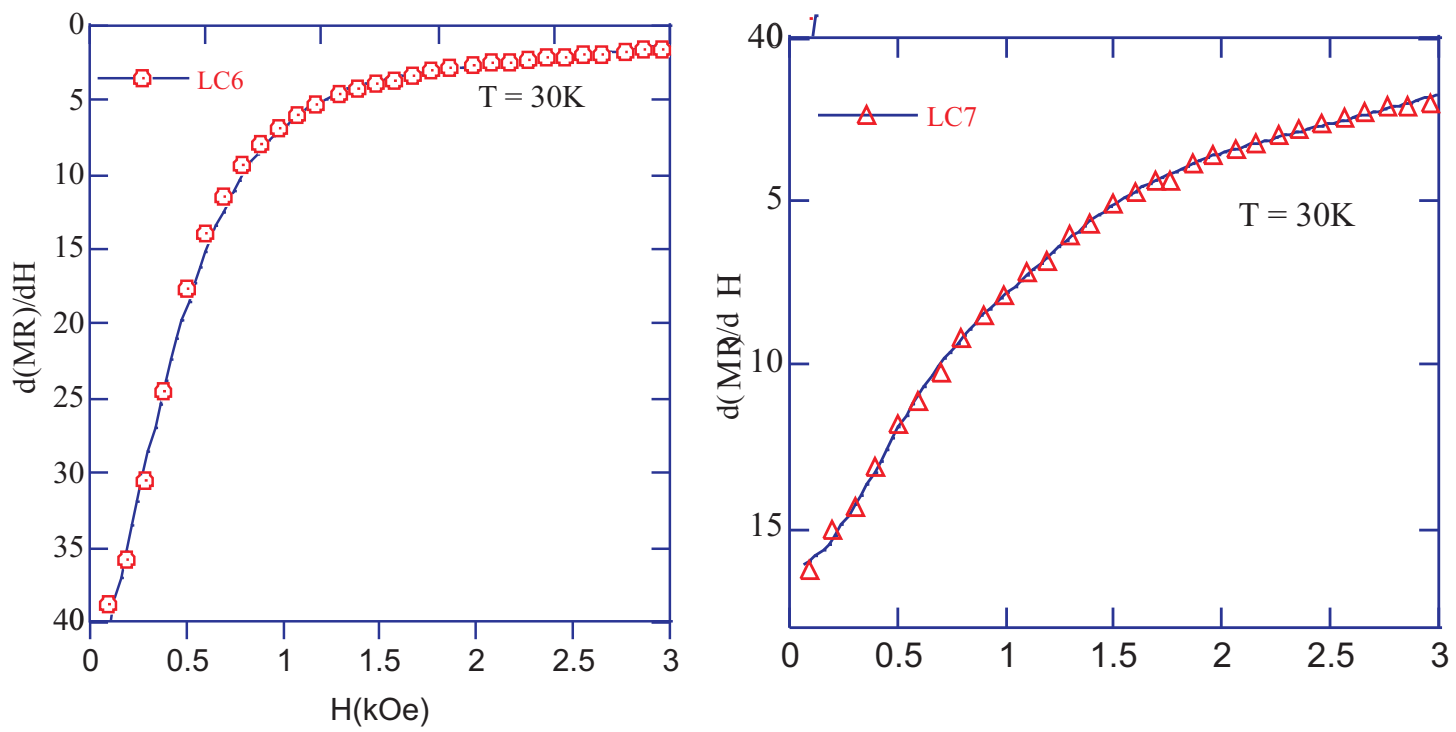

Fig. 8. Derivative of the experimental (MR-H) curve (dot) and the fitted curve (line) using Eq. (5) at $30 \mathrm{~K}$ in the magnetic field range of (0.2-3k Oe) for samples LC6 and LC7
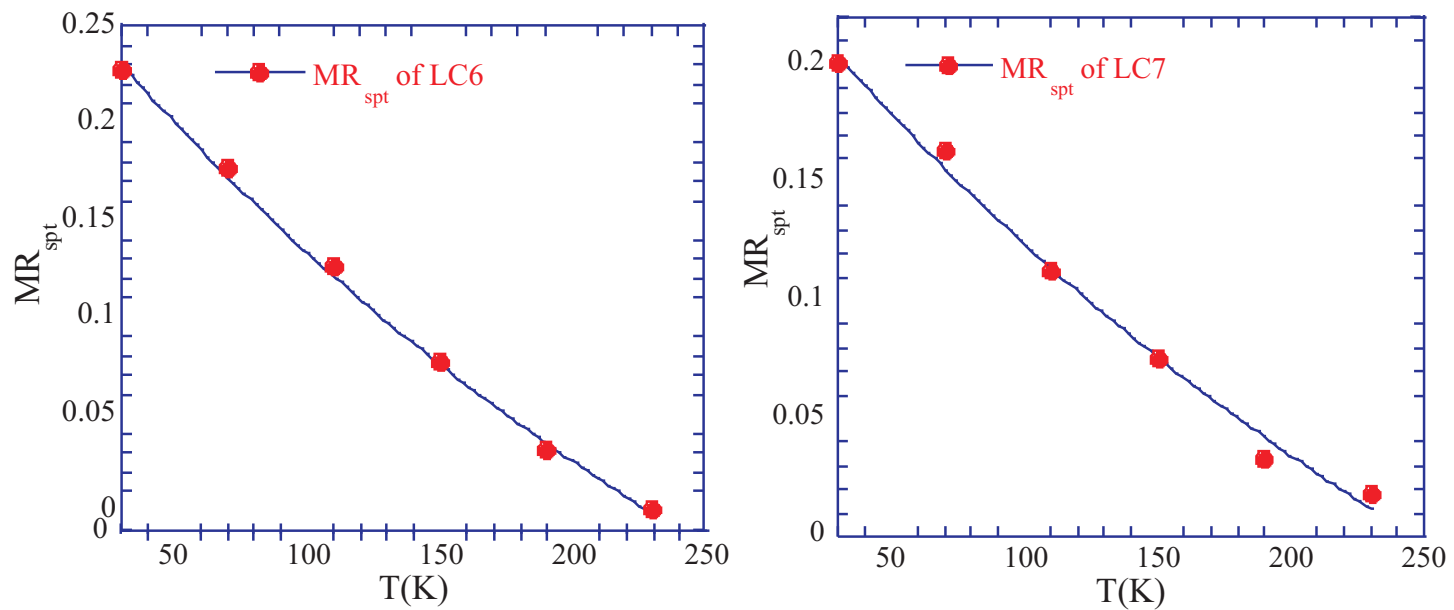

Fig. 9. The best fit of MRspt to a function of the form $a+b /(c+T)$ for samples LC6 with $a=-0.6653 ; b=514.3 \mathrm{~K} ; c=544.1 \mathrm{~K}$ and LC7 with $a=-0.5715$; $b=470.4 \mathrm{~K} ; c=576.4 \mathrm{~K}$

$\mathrm{T}_{C}$ of our system is much smaller. In this context we should note that the intergranular spin polarized tunneling have different temperature dependences for ferromagnetically coupled and superparamagnetically coupled grains [15]. 


\section{CONCLUSION}

In summary, we have studied the effect of sintering temperature on microstructure and low field magneto transport properties of polycrystalline LCMO. The ferromagenetic - paramagnetic (FM-PM) transition temperature remains almost constant, the metal insulation transition temperature shift towards lower temperatures as the particle size decreases. It has been found that LFMR increases as the sintering temperature (particle size) decreases but at the same time peak (intrinsic) MR decreases. This enhanced LFMR for small size particle is due to increased spin polarized tunneling behavior at lower temperature. We have analyzed our experimental MR data following a phenomenological model to separate out the MR arising from spin polarized transport, from the intrinsic contribution in our nanosize grannular LCMO samples. A detailed study on magnetic behavior and magnetoresistance properties with particle size is in progress and results will be forthcoming.

\section{ACKNOWLEDGEMENT}

This work has been sponsored by the Institute of Materials Science (IMS-VAST, Vietnam) and National Program on basic Research of Vietnam. The authors would like to thank Dr. Dao Nguyen Hoai Nam for valuable discussions.

\section{REFERENCES}

[1] E.Dagotto, T.Hotta, A.Moreo, Phys. Rep. 344 (2001) 1.

[2] C. Zener, Phys.Rev. 82 (1951) 403

[3] A. J. Millis, P. B. Littewood and B. Shraiman, Phys. Rep. Lett. 74 (1995) 5144

[4] H. Y. Hwang, S. W. Cheong, N. P. Ong, B. Batlogg, Phys. Rev. Lett. 77 (1996) 2041

[5] R. Mahesh, R. Mahendiran, A. K. Raychaudhuri, C. N. R. Rao, Appl.Phys.Lett. 68 (1996) 2291

[6] P. K. Siwach, R.Prasad, A.Gaur, H.K.Singh, G.D.Varma, O.N.Srivastava, J. Alloys and Compounds 443 (2007) 26

[7] R. D. Sánchez, J. Rivas, F. Rivas, C.Vázquez, M. A. Lopez-Quintela, M.T.Causa, M.Tovaz, S.B.Oseroff, Appl.Phys.Lett. 68 (1996) 134

[8] A.de Andrés, M.Garcia-Hernández and J.L.Martinez, Phys.Rev.B 60 (1999) 7328

[9] S. L. Yuan, J.Tang, L. Liu, W. Chen, L. F. Zhao, Y. Tian, H. Cao, G. H. Zhang, W. Feng, S. Liu, and Z. C. Xia, Europhys. Lett. 63 (2003) 433

[10] C.Vazquez-Vazquez, M.C.Blanco, M.A.Quintela-Lopez, R. D. Sanchez, J. Rivas and S.B.Oseroff, J. Mater. Chem. 8 (3) (1998) 991

[11] M. A. López-Quintela, L. E. Hueso, J. Rivas and F. Rivadulla, Nanotechnology 14 (2003) 212

[12] P. K. Siwach, D. P. Singh, H. K. Singh, N. Khare, A. K. Singh and O. N. Srivastas, J. Phys: Appl. Phys. 36 (2003) 1361

[13] P. Raychadhuri, K. Sheshadri, P.Taneja, S. Bandyopadhyay, P. Ayyub, A. K. Nigam, and R. Pinto, J. Appl. Phys. 84 (1998) 2048

[14] P. Dey and T. K. Nath, Phys. Rev. B73 (2006) 214425

[15] P. Raychadhuri, T. K. Nath, A. K. Nigam, and R. Pinto, Phys. Rev. B59 (1999) 13919.

Received 11 January 2008. 
